\title{
Practical Termination Strategies for Subcarrier Equalizer Tap Loading Algorithms
}

\author{
Alexander M. Wyglinski* Martin Cudnoch Fabrice Labeau Peter Kabal \\ Centre for Advanced Systems and Technologies in Communications (SYTACom) \\ Department of Electrical and Computer Engineering, McGill University \\ 3480 University Street, Montreal, QC, Canada H3A 2A7
}

\begin{abstract}
In this paper, a termination strategy for subcarrier equalizer tap loading algorithms is proposed. The objective of these algorithms is to non-uniformly distribute equalizer taps incrementally across the subcarriers of a multicarrier system to reduce the overall distortion introduced by the channel. Knowing when enough equalizer taps have been allocated is the task of the algorithm's termination strategy, which employs a number of criteria in the decision process. The proposed termination strategy, which is based on limiting the total number of taps used by the system, is presented and compared with three other strategies proposed earlier. Simulation results for a system employing the proposed strategy using different total tap limits emphasize the advantages of equalizer tap loading algorithms over conventional multicarrier equalization schemes.
\end{abstract}

Keywords: Multicarrier Modulation, Equalization, Adaptive Allocation

\section{Introduction}

To meet the demand for reliable high-speed wireless access, multicarrier modulation is becoming the transmission technology of choice for several applications, e.g., wireless local area network systems $[1,2]$. The primary advantage of multicarrier modulation is its ability to transmit information over frequency-selective fading channels using a "divide-andconquer" approach. Rather than transmitting data on a single carrier at a high data rate, information can be redistributed into several slower data streams, modulated on several different carriers, and transmitted simultaneously [3]. With respect to equalization and detection in multicarrier systems, the resulting receiver implementation complexity is lower since the frequency-selective fading channel has been transformed into a collection of approximately-flat fading subchannels.

There exists two fundamental types of equalizers that can be implemented in multicarrier transceivers: (i) time-domain equalizer or TEQ (a single equalizer located before the subcarriers separation of the received signal), (ii) per-tone equalizer or PTEQ (also referred to as a frequency-domain equalizer or FEQ). The disadvantage of employing a TEQ is that

This research was partially funded by the Natural Sciences and Engineering Research Council of Canada (NSERC) and Le Fonds "Nature et Technologies" du Québec (NATEQ).

*A. M. Wyglinski is now at the Information and Telecommunication Technology Center, The University of Kansas, Lawrence, KS, USA 66045-7612. it does not exploit the divide-and-conquer nature of multicarrier modulation, resulting in a very complex solution requiring many taps. This results in both high implementation complexity and power consumption. On the other hand, employing a PTEQ for each subcarrier fully exploits the channel spectrum transformation by the multicarrier transceiver. The solutions for a PTEQ can vary from a single tap PTEQ, employed in orthogonal frequency division multiplexing (OFDM) systems with a sufficient cyclic prefix and number of subcarriers [1], to multi-tap PTEQ designs [4]. Nevertheless, the savings in implementation complexity and power consumption is significant relative to TEQ solutions.

Although much work has gone into PTEQ design, there exists the potential to further increase the efficiency of an implementation with respect to hardware requirements and power consumption. In the case of single carrier systems, there exists several implementations where the tap lengths of the equalizer vary depending on some cost function or metric [5,6]. The idea of non-uniformly varying the PTEQ tap length across the subcarriers, using a subcarrier equalizer tap loading algorithm, has also been proposed in several designs $[7,8]$.

Deciding when an adequate number of taps have been allocated to each subcarrier is the task of the algorithm's termination strategy. Too many taps allocated will cause a reduction in distortion that does not justify the power consumption, whereas too few taps will give a poor performance. Therefore, the design of the termination strategy is important for an efficient implementation of the PTEQ. In this paper, we present a new termination strategy for subcarrier equalizer tap loading algorithms. Following a description of the multicarrier transceiver framework in Section 2 and a greedy subcarrier equalizer tap loading algorithm in Section 3, the proposed and three other termination strategies are presented in Section 4. Simulation results of multicarrier systems employing the proposed strategy is then presented in Section 5, followed by some concluding remarks in Section 6 .

\section{System Setup}

In this work, we employ a Modified Discrete Fourier Transform (MDFT) filterbank-based multicarrier system [9]. This type of multicarrier system was chosen for this work since the synthesis and analysis filters can be designed to be highly spectrally selective, and the intercarrier interference is minimized due to the phase offset between the real and imaginary data components. The general setup of this system, em- 


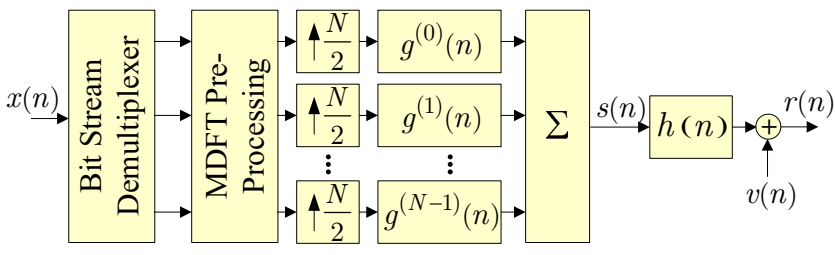

(a) Transmitter with channel.

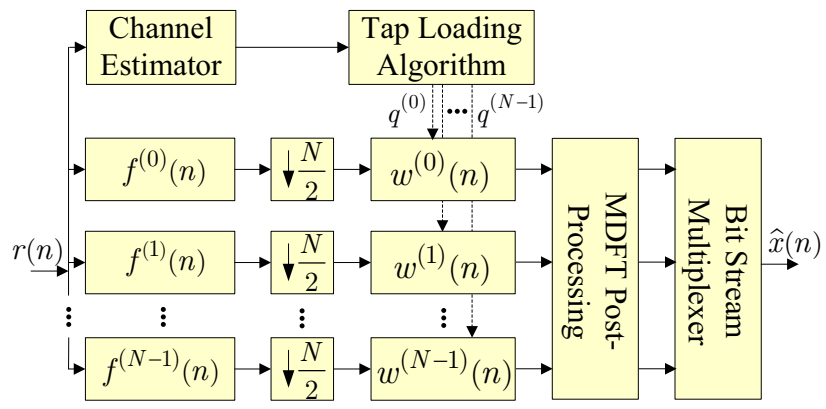

(b) Receiver with per-tone equalizers, channel estimator, and equalizer tap loading algorithm.

Fig. 1 Schematic of an MDFT multicarrier filterbank system performing subcarrier equalizer tap loading.

ploying a channel estimator, equalizer tap loading algorithm, and a bank or PTEQs, is shown in Fig. 1. The high speed complex input symbol stream, $x(n)$, is demultiplexed into $N$ streams, with stream $i$ having $b_{i}$ bits per symbol epoch. The bit streams are modulated onto one of several signal constellations consisting of $M_{i}=2^{b_{i}}$ points. The outputs $x^{(i)}(n)$, $i=0, \ldots, N-1$, are then MDFT pre-processed, where $x^{(i)}(n)$ is upsampled by a factor of 2 , the real and imaginary components are separated, one of the components is delayed by one sample, and the components are recombined. The outputs $y^{(i)}(n), i=0, \ldots, N-1$, are upsampled by a factor $N / 2$ and filtered by synthesis filters $g^{(i)}(n), i=0, \ldots, N-1$, before being summed together, yielding the composite transmit signal, $s(n)$. This signal is transmitted across the channel, where the multipath propagation and additive noise are modelled with channel impulse response $h(n)$ and noise $v(n)$. The received signal, $r(n)$, is separated into the $N$ subchannels using the analysis filters $f^{(i)}(n), i=0, \ldots, N-1$, and downsampled by a factor $N / 2$. The channel estimator extracts channel information from $r(n)$, which is then used by the equalizer tap loading algorithm to determine the number of equalizer taps for subcarrier $i, q^{(i)}$, for $i=0, \ldots, N-1$. Given the tap lengths, the subcarrier signals are equalized using $w^{(i)}(n)$, $i=0, \ldots, N-1$, MDFT post-processed, demodulated, and then multiplexed together to form the estimate $\hat{x}(n)$.

\section{Subcarrier Equalizer Tap Loading Algorithm}

The subcarrier equalizer tap loading algorithm used in this work is shown in Fig. 2. This loading algorithm operates in a "greedy" fashion [10] since it incrementally allocates an equalizer tap to the subcarrier that would yield the greatest decrease in the overall distortion.

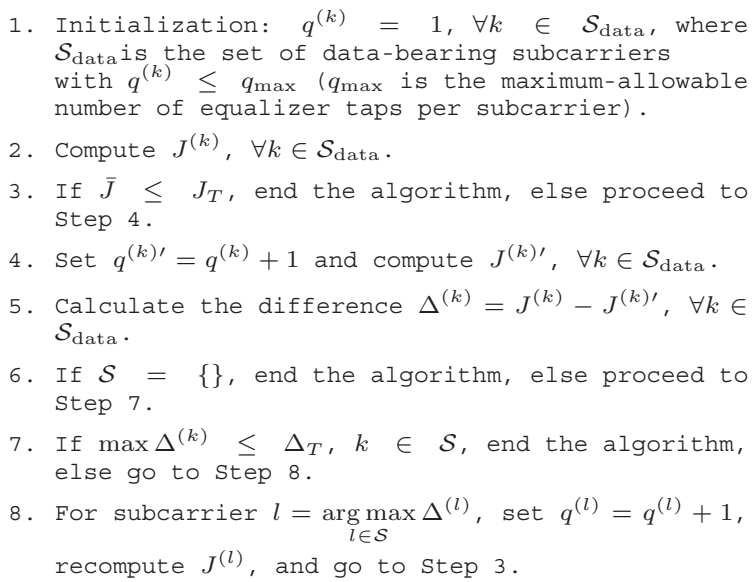

Fig. 2 Subcarrier equalizer tap loading algorithm employing a "greedy" approach [8].

Given a subset of subcarriers $\mathcal{S}_{\text {data }}$, which are data-bearing subcarriers, the algorithm begins by setting the lengths of the equalizers for the data-bearing subcarriers, $q^{(k)}, k \in \mathcal{S}_{\text {data }}$, to unit length. Using $q^{(k)}$, the optimal equalizer weights $w^{(k)}(n)$ are computed such that they minimize the distortion $J^{(k)}$ for each data-bearing subcarrier ${ }^{1}$. The arithmetic mean of the subcarrier distortion $J^{(k)}, \bar{J}$, is compared with the prescribed overall distortion threshold for the system, $J_{T}$. If $\bar{J} \leq J_{T}$, then none of the equalizer tap lengths need to be increased in order to reduce $\bar{J}$ and the algorithm ends. However, if $\bar{J}>J_{T}$, then the algorithm needs to increase some of the tap lengths $q^{(k)}$ in order to satisfy $\bar{J} \leq J_{T}$. In this case, the algorithm incrementally increases the length of the subcarrier equalizer that maximizes the decrease in $\bar{J}$. Therefore, the algorithm computes the equalizer weights $w^{(k) \prime}(n)$ and corresponding theoretical distortion values $J^{(k) \prime}$ when the lengths are $q^{(k) \prime}=q^{(k)}+1$ for all subcarriers belonging to $\mathcal{S}_{\text {data }}$. The differences $\Delta^{(k)}=J^{(k)}-J^{(k) \prime}$ are computed and the maximum difference is chosen from a set $\mathcal{S}$, where $\mathcal{S} \subseteq \mathcal{S}_{\text {data }}$ is the set of all subcarriers with allocations below the subcarrier tap limit, i.e., $q^{(k)} \leq q_{\max }$. If the set $\mathcal{S}$ is empty, which means that all data-bearing subcarriers have reached the maximum allocation of equalizer taps, $q_{\max }$, the algorithm finalizes the allocation and exits. Otherwise $\Delta^{(k)}, k \in \mathcal{S}$, is compared with the prescribed difference threshold $\Delta_{T}$. If $\max \left(\Delta^{(k)}\right) \leq \Delta_{T}$, then the algorithm breaks out and ends since the largest difference in distortion is considered negligible. If $\max \left(\Delta^{(k)}\right)>\Delta_{T}$, then the algorithm chooses subcarrier $l, l \in \mathcal{S}$, which has the largest $\Delta^{(k)}$. The algorithm updates $q^{(l)}=q^{(l)}+1$ then computes $J^{(l)}$. Finally, $\bar{J} \leq J_{T}$ is compared and the process repeats.

\footnotetext{
${ }^{1}$ Assuming the bit error rate (BER) is monotonically related to the distortion, this would also minimize the BER. As a result, the distortion is used as a metric of performance in this algorithm for convenience.
} 


\section{Termination Strategies}

The loading algorithm in Section 3 and Fig. 2 has three steps (Steps 3, 6, and 7) that are designed to end the algorithm once some prescribed criterion has been satisfied. Each of these steps forms a termination strategy of the algorithm, and are partially responsible for the adequate allocation of taps. These strategies can be designed to end the tap loading process for either a single subcarrier or the entire system. In this section, we propose a termination strategy based on limiting the total number of taps allocated, and briefly study three other termination strategies.

\subsection{Mean Cost Function-Based Strategy}

This termination strategy, employed in Step 3 of Fig. 2, ends the loading algorithm once an adequate number of taps have been allocated such that the overall distortion is below some predefined limit. This is a global strategy since it requires information from all the subcarriers in order to make a decision and end the algorithm. Thus, the strategy ends the loading algorithm when

$$
\bar{J} \leq J_{T}
$$

since the mean cost function is above the minimum-allowable level of quality for the transmission.

This strategy requires some advance knowledge of the channel and noise level in order to set $J_{T}$ to an appropriate value. If $J_{T}$ is below the noise floor, the loading algorithm will never satisfy Eq. (1) irrespective of the number of taps, i.e., the algorithm will never end. Conversely, if $J_{T}$ is set too high, the algorithm will end before an adequate number of taps have been allocated.

\subsection{Mean Cost Function Difference-Based Strategy}

This termination strategy, employed in Step 7 of Fig. 2, operates by ending the entire allocation process whenever the largest difference between the current and subsequent subcarrier cost function is below some prescribed threshold. Therefore, this is a global strategy since it also requires information from all the subcarriers in order to make a decision and end the algorithm. The rationale behind this strategy is that the tap loading ends when the distortion decrease does not warrant an increase in implementation complexity. This strategy is equivalent to comparing the largest difference of the current and subsequent mean cost functions to some prescribed limit ${ }^{2}$.

Mathematically, this strategy terminates the loading algorithm when

$$
\bar{\Delta}=\frac{\bar{J}-\bar{J}^{\prime}}{\bar{J}} \leq \Delta_{T}
$$

where $\bar{J}$ and $\bar{J}^{\prime}$ are the current and subsequent mean cost functions, and $\Delta_{T}$ is the prescribed mean cost function difference limit.

The advantage of using this strategy is that a percentage metric is employed rather than an absolute metric. As a result,

\footnotetext{
${ }^{2}$ The subsequent allocation is generated by adding an equalizer tap to the current allocation to the subcarrier yielding the largest change in the cost function.
}

prior knowledge of the channel conditions and system implementation are not required in choosing an appropriate value for $\Delta_{T}$. However, this strategy does not take into account the practical restrictions imposed by a hardware implementation, such as a limit on the amount of available memory or processing power. If the threshold $\Delta_{T}$ is high, fewer taps will be allocated, resulting in the system's hardware resources being underused. On the other hand, a low value for $\Delta_{T}$ will result in longer equalizers, requiring more hardware to implement. These issues will be addressed in the next two subsections.

\subsection{Maximum Taps per Subcarrier-Based Strategy}

Instead of ending the loading algorithm when some global criterion has been met, it may be more advantageous to stop allocating taps to specific subcarriers by limiting the individual subcarrier tap lengths. For instance, in Section 3, the maximum number of taps per subcarrier is limited to be below $q_{\text {max }}$. If any of the subcarriers have reached $q_{\max }$, they would no longer be eligible to receive additional taps. Although this strategy indirectly has a global effect ${ }^{3}$, this strategy only looks at the tap length of a specific subcarrier and does not require information from other subcarriers. An advantage of this strategy is that the maximum lengths of equalizers are known in advance, thus the hardware design can employ a fixed amount of resources for the equalizer implementation. Moreover, the maximum possible group delay of the equalizers will also be known. Finally, with an increase in the length of an equalizer, there is linear increase in the number of computations. Thus, a hard limit on the equalizer length establishes an upper bound on both the total delay and the total number of operations required. The only disadvantage of this strategy is that it can only be used in combination with a second termination strategy. Otherwise, all equalizers would be of length $q_{\max }$.

\subsection{Proposed Strategy: Bank of Equalizer Taps}

The proposed termination strategy builds upon the strategy in Section 4.3 by imposing constraints on the maximum complexity of the hardware implementation. However, instead of limiting the number of taps per subcarrier, the proposed strategy limits the total number of equalizer taps allocated. Thus, for the same hardware complexity the loading algorithm has additional flexibility in allocating taps.

A finite pool of equalizer taps of size $q_{\mathrm{tot}}$ is available to the loading algorithm when allocating across the data-bearing subcarriers of the system. Every time the length $q^{(k)}$ of a subcarrier equalizer is incremented, the pool of available taps is decremented. Once the pool is empty, no more taps are available and the algorithm terminates. In other words, the algorithm continues to allocate until

$$
\sum_{k \in \mathcal{S}_{\text {data }}} q^{(k)}=q_{\text {tot }},
$$

in which case the algorithm ends. The only disadvantage of this strategy is that the worst case group delay of the subcarrier

\footnotetext{
${ }^{3}$ In Step 6 of Fig. 2, the algorithm ends when all subcarriers have each reached the tap limit $q_{\max }$.
} 


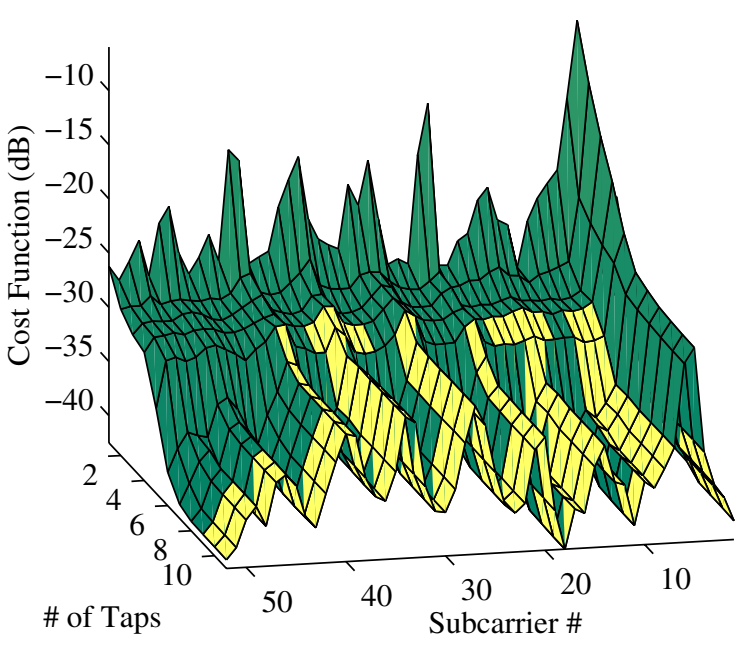

Fig. 3 Subcarrier MMSE as function of subcarrier equalizer length using the ETSI HiperLAN/2 Channel B at an SNR of $98 \mathrm{~dB}$.

equalizers is larger relative to the strategy in Section 4.3. On the other, the greater degree of flexibility gained by the loading algorithm can result in allocations with a smaller overall distortion.

\section{Simulation Results}

Using the ETSI HiperLAN/2 indoor channel models [11], we employ the proposed algorithm in an MDFT filterbank multicarrier system [9]. The synthesis and analysis filters are modulated versions of a root raised cosine lowpass filter. Many of the operating parameters of the system correspond to the IEEE Std. 802.11a [1], including the modulation schemes (BPSK, QPSK, square 16-QAM, and square 64-QAM modulation), subcarrier spacing, and operating frequency $(5 \mathrm{GHz})$. For straightforward comparison, the system did not employ channel coding. Minimum mean squared error (MMSE) equalizers were employed per subcarrier and the subcarrier MMSE distortion, defined in [8], was employed as the subcarrier cost function. Since the system is operating in an indoor environment, the channel is quasi-stationary. Therefore, the channel is assumed to be time-invariant over a sufficiently long period of time and that the equalizer weights and lengths need to be determined once for a specific channel. Moreover, we assume the channel is perfectly known at the receiver, although in practice data-aided channel estimation techniques would be employed.

The subcarrier equalizer tap loading algorithm from Fig. 2 was employed in this work, with Step 3 changed to the proposed termination strategy in Section 4.4. The termination strategies of Steps 6 and 7 were also included in the loading algorithm. As a result, we chose $q_{\max }=20$ taps and $\Delta_{T}=10^{-4}$. We also investigated the performance of the algorithm when $q_{\mathrm{tot}}=104$ taps and $q_{\mathrm{tot}}=520$ taps. Finally, all results were obtained via Monte Carlo simulations, where simulations ended after either the subcarrier with the fewest

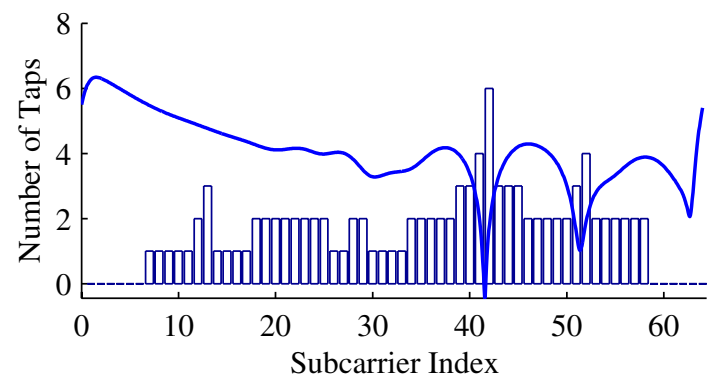

(a) Equalizer tap allocation at an SNR of $31.7 \mathrm{db}$.

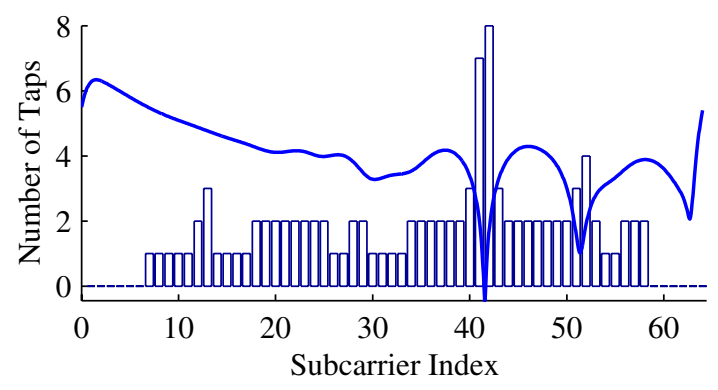

(b) Equalizer tap allocation at an SNR of $38.7 \mathrm{db}$.

Fig. 4 Equalizer tap allocation given the ETSI HiperLAN/2 Channel A (superimposed) for different SNR values and $q_{\text {tot }}=104$ taps.

errors accumulated 100 errors or the overall accumulated error equaled $N \times 1000$.

\subsection{Cost Function Versus Number of Equalizer Taps}

The subcarrier MMSE distortion as a function of the number of equalizer taps is shown in Fig. 3 for all 52 data-bearing subcarriers given the ETSI HiperLAN/2 Channel B at an SNR of $98 \mathrm{~dB}$. Note the the dark regions of the surface plot indicate the equalizer tap allocation given the channel and SNR value. The results show that the MMSE decreases monotonically as the number of equalizer taps increases. However, the MMSE versus the number of equalizer taps is not a strictly convex function for all subcarriers since the equalizer taps are constrained to uniform sampling locations, which might not correspond to an optimal solution. Therefore, each additional tap to a subcarrier equalizer may result in a decrease in distortion. However, that decrease may not be smaller than the previous allocations for that subcarrier.

During our simulations it was noticed that for the small bank of $q_{\mathrm{tot}}=104$ taps, the limiting factor of the loading algorithm was the size of the bank of equalizer taps. This translates into an early stop in the allocation. Conversely, when $q_{\mathrm{tot}}=520$ taps is used, the $\Delta_{T}$ limit will be the prevalent mode of termination for the loading algorithm. As a result, the algorithm ends when a substantial number of taps have been allocated. Finally, the subcarrier tap limit was seldom employed (e.g., three subcarriers out of all the data-bearing subcarriers for all five ETSI channels ever reached that limit). 


\subsection{Subcarrier Tap Allocation}

Several observations can be made regarding the equalizer tap allocations for two different SNR values, such as allocations at 31.7 and $38.7 \mathrm{~dB}$ given the ETSI HiperLAN/2 Channel A, as shown in Fig. 4. First, when a spectral null is present, the loading algorithm will try to add more equalizer taps to invert the effects of the distortion in that subcarrier. However, if the spectral null is too steep and the incremental gain of additional taps on the cost function is small, the algorithm will stop allocating taps to that subcarrier. This effect can be seen in Fig. 4, where subcarrier 42 is in the vicinity of a spectral null. In this case, the algorithm stops allocating taps to the subcarrier after only two taps.

When $q_{\mathrm{tot}}=520$ taps, the number of taps allocated increases relative to an increase in the SNR. Moreover, in several subcarriers, the tap lengths may also increase. This is due to the lowering of the noise floor, leaving the frequencyselective fading channel as the predominant form of distortion. However, when $q_{\text {tot }}=104$ taps, it is observed that the tap allocations are rearranged at different SNR values. For instance, several subcarriers around Subcarrier 42 may loose taps while others gain taps after an increase in SNR. This rearrangement occurs when the distortion versus tap number function is not convex, due to the suboptimal placement of taps in an equalizer.

\subsection{BER Results}

The BER results for a multicarrier system employing 64QAM modulation given ETSI HiperLAN/2 Channel E are presented in Fig. 5. At low SNR values, tap allocation is ineffective when the noise floor is high ${ }^{4}$. This means that the distortion versus tap number function will flatten out early in the curve and that additional taps will not improve performance. As a result, not many taps are allocated. As the SNR increases, more taps are allocated as the noise floor lowers. Consequently, the channel becomes the principal source of distortion, which can be handled by the equalizers more effectively. Therefore, more equalizer taps are employed since their addition significantly improves the overall performance of the system. Moreover, with more equalizer taps available to the loading algorithm, the system employing $q_{\text {tot }}=520$ taps has drastically better BER performance relative to a system with $q_{\text {tot }}=104$ taps at high SNR values starting at $20 \mathrm{~dB}$. Finally, relative to systems using uniform-tap length subcarrier equalizers with an equivalent total number of taps, employing a rounded average number of taps per equalizer, the system employing tap loading algorithms performs better.

\section{Conclusion}

Several termination strategies for equalizer tap loading algorithms have been presented, including a proposed strategy aimed at limiting the total number of taps. Employing several of these strategies together, including the proposed strategy,

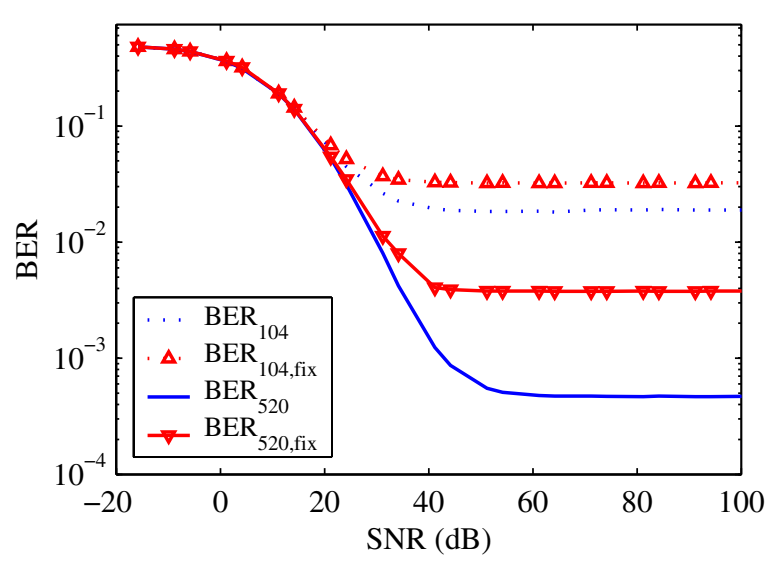

Fig. 5 BER curves for fixed tap length (with triangular markers) and tap-allocated (without triangular markers) multicarrier systems employing square 64-QAM modulation across all subcarriers given the ETSI Channel E. Results are shown for $q_{\text {tot }}=104$ taps (dotted lines) and $q_{\text {tot }}=520$ taps (solid lines).

the error robustness of the system is enhanced, especially at medium to high SNR values. Moreover, a decrease in the total number of taps available to the system will result in a substantial decrease in error robustness.

\section{References}

[1] IEEE Std. 802.11a, "Wireless LAN medium access control (MAC) and physical layer (PHY) specifications: High-speed physical layer in the $5 \mathrm{GHz}$ band," Nov. 1999.

[2] ETSI TS 101 475, "Broadband radio access networks (BRAN): HIPERLAN type 2; physical (PHY) layer,” Dec. 2001.

[3] J. Louveaux, Filter Bank Based Multicarrier Modulation for xDSL Transmission. $\mathrm{PhD}$ thesis, Université Catholique de Louvain, Louvainla-Neuve, Belgium, May 2000.

[4] K. Van Acker, G. Leus, M. Moonen, O. Van de Wiel, and T. Pollet, "Per tone equalization for DMT-based systems," IEEE Trans. Commun., vol. 49, pp. 109-119, Jan. 2001.

[5] Y. Gong and C. F. N. Cowan, "A novel variable tap-length algorithm for linear adaptive filters," in Proc. IEEE Int. Conf. Acoust., Speech, Signal Process., vol. 2, (Montreal, QC, Canada), pp. 825-828, May 2004.

[6] F. Riera-Palou, J. M. Noras, and D. G. M. Cruickshank, "Variable length equalizers for broadband mobile systems," in Proc. 52nd IEEE Veh. Technol. Conf. - Fall, vol. 5, (Boston, MA, USA), pp. 2478-2485, Sept. 2000.

[7] A. M. Wyglinski, P. Kabal, and F. Labeau, "Adaptive filterbank multicarrier wireless systems for indoor environments," in Proc. 56th IEEE Veh. Technol. Conf. - Fall, vol. 1, (Vancouver, BC, Canada), pp. 336340, Sept. 2002.

[8] A. M. Wyglinski, P. Kabal, and F. Labeau, "Variable-length subcarrier equalizers for multicarrier systems," in Proc. 60th IEEE Veh. Technol. Conf. - Fall, (Los Angeles, CA, USA), Sept. 2004.

[9] T. Wiegand and N. J. Fliege, "Equalizers for transmultiplexers in orthogonal multiple carrier data transmission," in Proc. Euro. Signal Processing Conf., vol. 2, (Trieste, Italy), pp. 1211-1214, Sept. 1996.

[10] A. Gersho and R. M. Gray, Vector Quantization and Signal Compression. Communications and Information Theory, Kluwer Academic Publishers, 1992.

[11] J. Medbo and P. Schramm, "Channel models for HIPERLAN/2 in different indoor scenarios," ETSI EP BRAN Doc. No. 3ERI085B, European Telecommunications Standards Institute, 1998.

\footnotetext{
${ }^{4}$ The influence of the noise floor on the system's BER performance is partially dependent on the subcarrier modulation scheme employed.
} 